Shigeo Kure • Yoshiyuki Sakata • Shigeaki Miyabayashi

Kazutoshi Takahashi · Toshikatsu Shinka

Yoichi Matsubara • Hisao Hoshino • Kuniaki Narisawa

\title{
Mutation and polymorphic marker analyses of 65K- and 67K-glutamate decarboxylase genes in two families with pyridoxine-dependent epilepsy
}

Received: November 4, 1997 / Accepted: December 5, 1997

\begin{abstract}
Pyridoxine-dependent epilepsy is a disease inherited as an autosomal recessive trait, characterized by rapid response to pharmacological dosages of pyridoxine. The defect has been suggested to reside in glutamate decarboxylase (GAD), since a mutant GAD with an abnormally high $K_{\mathrm{m}}$ for a cofactor, pyridoxal phosphate, could not synthesize an adequate amount of $\gamma$-amino butyric acid [Scriver and Whelan (1969) Ann NY Acad Sci 166: 83]. To test this hypothesis, we studied two affected families by screening for mutations in the GAD mRNA and by analyzing a polymorphic marker in the $G A D$ gene. Since two forms of GAD, GAD65 and GAD67, have been identified in human brain, we analyzed both forms. To overcome the limited accessibility of brain tissues, we utilized the minute amounts of GAD mRNAs ectopically transcribed in lymphoblasts. The ectopic GAD transcripts were amplified by reversetranscription-mediated, nested polymerase chain reaction for mutation analysis. Two and three base substitutions were found in GAD65 and GAD67 cDNAs, respectively. All of them were, however, polymorphisms that were also found in control subjects. We then examined a (CA) repeat polymorphism in the GAD65 gene and found that different maternal alleles were transmitted to two affected sibs in one family. Thus, an etiological mechanism other than a $K_{\mathrm{m}}$ mutant GAD is responsible for pyridoxine-dependent epilepsy.
\end{abstract}

Key words cDNA sequence $\cdot K_{\mathrm{m}}$ mutant $\cdot$ Familial analysis $\mathrm{CA}$ repeat $\cdot$ Ectopic transcripts

S. Kure $(\varangle) \cdot$ Y. Sakata $\cdot$ K. Takahashi $\cdot$ T. Shinka $\cdot$ Y. Matsubara K. Narisawa

Department of Biochemical Genetics, Tohoku University School of Medicine, 1-1 Seiryo-machi, Aoba-ku, Sendai 980-8574, Japan

Tel. +81-22-717-8140; Fax +81-22-717-8142

e-mail: skure@mail.cc.tohoku.ac.jp

S. Miyabayashi

Department of Pediatrics, Tohoku University School of Medicine, Sendai, Japan

H. Hoshino

Department of Pediatrics, Hitachi General Hospital, Hitachi, Japan

\section{Introduction}

Pyridoxine-dependent epilepsy was first reported as a specific form of neonatal epilepsy (Hunt et al. 1954), and is characterized by generalized seizures that are refractory to the standard anticonvulsants, but respond to pharmacological doses of pyridoxine (Scriver and Gibson 1995). The dependency is permanent. If daily oral supplementation with pyridoxine is interrupted, seizures recur within several days. This is an inherited disease with an autosomal recessive trait (MIM266100). The gene responsible for this genetic disease is unknown. Scriver and Whelan (1969) suggested an etiological hypothesis with respect to a mutant glutamate decarboxylase (GAD: EC4.1.1.15), a $\gamma$-amino butyric acid (GABA) synthesizing enzyme; a mutation in the $G A D$ gene would create a mutant form of GAD with an abnormally high $K_{\mathrm{m}}$ for its cofactor pyridoxal phosphate (PLP). This mutation has been widely cited as the most likely etiology for this disorder. To verify the hypothesis, the enzymatic activities of GAD were examined in kidney (Yoshida et al. 1971), frontal cortex (Lott et al. 1978), and cultured skin fibroblasts (Gospe et al. 1994) from patients. The interpretations of these results were, however, still moot (Scriver and Gibson 1995). These circumstances prompted us to undertake the molecular analysis of the GAD.

This study was designed to demonstrate the existence of a kinetic mutant of GAD by screening for mutations in the GAD mRNA of the patients. Since two forms of GAD, GAD65 and GAD67, are expressed in brain, we analyzed both isozymes (Bu et al. 1992; Bu and Tobin 1994). To overcome the limited accessibility of brain tissue from the patients, we used illegitimate GAD mRNA in the lymphoblasts. Illegitimate mRNA corresponds to the very low levels of transcript of a specific gene in nonspecific cells (Sarkar and Sommer 1989; Takahashi et al. 1992). The GAD65 and GAD67 cDNA fragments were amplified for direct sequence analyses. Another approach used in this study was allele transmission analysis with a polymorphic marker which we recently identified in the GAD65 gene 
(Kure et al. 1997). These two molecular approaches enabled us to test the etiological hypothesis for pyridoxinedependent epilepsy.

\section{Patients and methods}

Patients. Two affected families (A and B) showed typical responses to pyridoxine treatment. Family A had two affected children, a brother and a sister. The case history of the brother was previously described (Iinuma et al. 1971) and the clinical symptoms of the brother and the sister were similar. In family B, a healthy sister and an affected boy were born to nonconsanguineous parents. The patient first had generalized convulsions at 9 hours of age. At $53 \mathrm{~h}$ after birth, the patient received an intravenous administration of $20 \mathrm{mg}$ pyridoxine and the seizures disappeared within $3 \mathrm{~min}$. To confirm the diagnosis of pyridoxine-dependent epilepsy, pyridoxine administration was discontinued when he was 6 months old. Generalized convulsions developed within 3 days. He was placed on oral pyridoxine therapy again and had neither convulsions nor developmental delay, but a mild weakness of muscle tone.

Direct sequence analysis of GAD65 and GAD67 cDNA fragments. Procedures for cDNA synthesis from lymphoblast cells were previously reported (Kure et al. 1992). The sequences of primers used in this study are shown in Table 1. The entire coding regions of the GAD65 and GAD67 cDNA were amplified in the first round PCR with
HGAD $2 \times 8$ and HGAD $21 \times 23$, respectively. Seven and twelve overlapping cDNA fragments were amplified in second-round PCR for GAD65 and GAD67, respectively. The primer sets for the GAD65 cDNA were HGAD $1 \times 13$, $9 \times$ $13,10 \times 13,3 \times 14,11 \times 14,5 \times 15$, and $12 \times 15$, while those for the GAD67 cDNA were HGAD $24 \times 47,54 \times 47,43 \times$ $47,55 \times 47,44 \times 14,56 \times 14,45 \times 14,57 \times 14,5 \times 16,58$ $\times 16,46 \times 16$, and $59 \times 16$. The sequence of M13 reverse primer was added to the $5^{\prime}$ ends of the forward primers, and the reverse primers were 5 -labelled with biotin (Table 1 ). Single-strand templates were prepared with avidin-coated magnetic beads (Dynal, Oslo, Norway), and subjected to dideoxy sequencing analysis with a fluorescence-labelled M13 reverse primer and an A.L.F. DNA Sequencer (Pharmacia, Uppsala, Sweden).

Polymorphic marker analysis. Polymorphic patterns of a (CA)-repeat region in the GAD65 gene were identified as previously reported (Kure et al. 1997). Fluorescencelabeled DNA fragments were used as the size standards (Applied Biosystems, Foster City, CA, USA).

\section{Results}

Amplification and sequence analysis of the GAD65 cDNA fragments

The GAD65 cDNA was amplified from the ectopic GAD mRNA in lymphoblasts by reverse-transcription-mediated, nested PCR. Seven overlapping GAD65 cDNA fragments

Table 1 The polymerase chain reaction (PCR) primers used in this study

\begin{tabular}{|c|c|}
\hline Name & Sequence $^{1}$ \\
\hline HGAD 1 & 5'-AGGAAACAGCTATGACAAAGCCGATGGCATCTCCG \\
\hline HGAD 2 & 5'-AGGCGACCTGCTCCAGTCT \\
\hline HGAD 3 & 5'-GGAAACAGCTATGACTCTGGCGATGGGATATTTTC \\
\hline HGAD 5 & 5'-AGGAAACAGCTATGACGTGGAATCCACACAAGATG \\
\hline HGAD 8 & 5'-TGTGACAACTTAATTGTCTACCTAG \\
\hline HGAD 9 & 5'-AGGAAACAGCTATGACGCAGCTGCTCCAAAGTGG \\
\hline HGAD 10 & 5'-GGAAACAGCTATGACGGGCATCCTAGATACTTCAATC \\
\hline HGAD 11 & 5'-AGGAAACAGCTATGACGATTCCAТCTGAТCTТGAAAG \\
\hline HGAD 12 & 5'-GGAAACAGCTATGACAAGGATATGAGATGGTGTTTGA \\
\hline HGAD 13 & $5^{\prime}$-BABTGCTGTACATGTTGGATATGGC ${ }_{2}^{2}$ \\
\hline HGAD 14 & 5'-BABGAGAATGGCAGAGCACTGCAA ${ }^{2}$ \\
\hline HGAD 15 & 5'-BABGTGGAACAGCTTGGTGAGCA ${ }^{2}$ \\
\hline HGAD 16 & 5'-BABAACTCATGTTCTGCGAAGGATG ${ }^{2}$ \\
\hline HGAD 21 & 5'-GAGGACTCTGGACAGTAGAG \\
\hline HGAD 23 & 5'-TTTGTTCTGGAGTGCCAGAGG \\
\hline HGAD 24 & 5'-CAGGAAACAGCTATGACGACCGAGCTGATGGCG \\
\hline HGAD 43 & 5'-CAGGAAACAGCTATGACTAGAGATCTGCTTCCGGC \\
\hline HGAD 44 & 5' -CAGGAAACAGCTATGACATTGCACCAGTGTTTG \\
\hline HGAD 45 & 5'-CAGGAAACAGCTATGACAGGGGGAAAATAATTCC \\
\hline HGAD 46 & 5' -AGGAAACAGCTATGACAGAGAAGAATTTGAGATGG \\
\hline HGAD 47 & 5'-ВАТСТСТСТСАТСТTСТTAAGTGT ${ }^{2}$ \\
\hline HGAD 54 & 5' -CAGGAAACAGCTATGACTTCTTGCAAAGGACCAAC \\
\hline HGAD 55 & 5'-CAGGAAACAGCTATGACCAGATCCTGGTTGACTG \\
\hline HGAD 56 & 5' -CAGGAAACAGCTATGACTTCCCGGAAGTTAAGAC \\
\hline HGAD 57 & 5' -CAGGAAACAGCTATGACCTTTGGTTGCATGTCG \\
\hline HGAD 58 & 5'-CAGGAAACAGCTATGACAAGGCAATTCAGTGTGGC \\
\hline HGAD 59 & 5' -CAGGAAACAGCTATGACGATGGAGTCAGGTACGAC \\
\hline
\end{tabular}

${ }^{1}$ The sequence in bold is the annealing site for the M13 reverse sequencing primer.

${ }^{2} \mathrm{~B}$ stands for a nucleotide conjugated with biotin. 
contained the whole protein-coding region of GAD65 cDNA. No difference in size was observed among the amplified cDNA fragments from the patients and controls. Direct sequence analysis revealed two homozygous nucleotide substitutions in all patients examined, T790C and T1597A, both of which caused no amino acid change. Both T790C and T1597A substitutions were also observed in five control lymphoblast cells in a homozygous form.

Amplification and sequence analysis of GAD67 cDNA fragments

Twelve overlapping GAD67 cDNA fragments were amplified. Direct sequence analysis of each GAD67 cDNA fragment revealed three base substitutions, C661T, G753A, and T1852C. The G753A and T1852C were found in a homozygous form in all three patients, while the C661T substitution was detected in a heterozygous form in all the patients. Both C661T and T1852C substitutions caused no amino acid substitution, while the $\mathrm{G} 753 \mathrm{~A}$ resulted in an amino acid replacement, of arginine by lysine, at amino acid position 68. All three substitutions were, however, also found in five Japanese control subjects.

Analysis of a polymorphic marker in the GAD65 gene

To elucidate a transmission profile of each GAD65 gene allele in the affected family $\mathrm{A}$, we examined the patterns of CA repeat polymorphism in intron 15 of the GAD65 gene (Kure et al. 1997). Observed alleles for the father, the mother, the affected brother, and the affected sister were (21 repeats, 27 repeats), $(20,25),(21,25)$, and $(20,21)$,

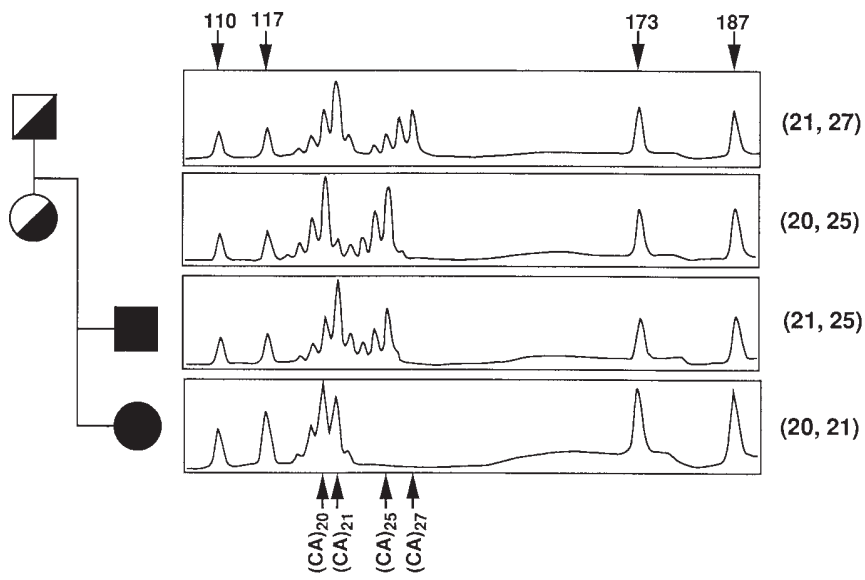

Fig. 1 Transmission analysis of the GAD65 gene allele with a (CA)repeat polymorphism. A polymorphic (CA)-repeat region was amplified by polymerase chain reaction (PCR) with a fluorescent-labeled primer. The electrophoresis profiles of the PCR products were printed out with a computer software program, Fragment Manager (Pharmacia). The numerical figures on the top of the figure indicate the length of the DNA size markers, respectively. The two numbers in parentheses represent a pair of GAD65 gene alleles for each individual respectively (Fig. 1). Both of the affected sibs inherited the allele of 21 repeats from their father, whereas they inherited different maternal alleles. The brother inherited the allele of 25 repeats, whereas the sister inherited the allele of 20 repeats.

\section{Discussion}

We studied the entire protein-coding regions of both GAD65 and GAD67 mRNAs from patients with pyridexine $\mathrm{B} 6$ dependent epilepsy. If there were a mutant GAD65 or GAD67 with an abnormal $K_{\mathrm{m}}$ for PLP, a mutant GAD mRNA with an altered protein-coding region would need to exist. Five nucleotide changes were found; two in GAD65 cDNA and three in GAD67 cDNA. Four of them caused no amino acid change, while one base change in the GAD67 mRNA, G753A, caused a basic amino acid substitution from arginine to lysine at amino acid position 68 . Homozygotes of G753A were, however, found among the control subjects, suggesting that G753A was not a mutation responsible for pyridoxine-dependent epilepsy, but a polymorphism commonly observed in Japan. Four other silent base changes were also found in the control subjects. Thus far, there is no evidence that altered forms of GAD65 or GAD67 mRNA existed in the cells from patients with pyridoxine-dependent epilepsy.

Based on the allele transmission analysis, it seems unlikely that the GAD65 gene is responsible for pyridoxine dependency. The analysis of (CA)-repeat polymorphism in the GAD65 gene suggested that the two affected sibs of family A inherited the different maternal alleles of the $G A D 65$ gene. If the $G A D 65$ gene were responsible for pyridoxine-dependent epilepsy, this result would serve to contradict the clinical observation that pyridoxinedependent epilepsy is inherited as an autosomal recessive trait. We attempted to find a (CA) repeat region in the $G A D 67$ gene by the same method but failed. Since two affected sibs in family $\mathrm{A}$ had the same pattern of three exonic polymorphisms found in the GAD67 gene, C661T, $\mathrm{G} 753 \mathrm{~A}$, and $\mathrm{T} 1852 \mathrm{C}$, we could not exclude the GAD67 gene from the candidate pool of genes by polymorphic marker analysis alone.

Taken together, these results suggest that an etiological mechanism other than a $K_{\mathrm{m}}$ mutant GAD may operate in pyridoxine-dependent epilepsy.

Acknowledgments This work was supported by a grant from the Ministry of Education, Science, and Culture and the Ministry of Health and Public Welfare, Japan.

\section{References}

Bu DF, Tobin AJ (1994) The exon-intron organization of the genes (GAD1 and GAD2) encoding two human glutamate decarboxylases (GAD67 and GAD65) suggests that they derive from a common ancestral GAD. Genomics 21: 222-228

Bu DF, Erlander MG, Hitz BC, Tillakaratne NJ, Kaufman DL, Wagner-McPherson CB, Evans GA, Tobin AJ (1992) Two human 
glutamate decarboxylases, 65-kDa GAD and 67-kDa GAD, are each encoded by a single gene. Proc Natl Acad Sci USA 89: 21152119

Gospe SM Jr, Olin KL, Keen CL (1994) Reduced GABA synthesis in pyridoxine-dependent seizures. Lancet 343: 1133-1134

Hunt A, Stokes J, McCrory W, Striud H (1954) Pyridoxine dependency: Report of a case of intractable convulsions in an infant controlled by pyridoxine. Pediatrics 13: 140-143

Iinuma K, Narisawa K, Yamauchi N, Yoshida T, Mizuno T (1971) Pyridoxine dependent convulsion: effect of pyridoxine therapy on electroencephalograms. Tohoku J Exp Med 105: 19-26

Kure S, Takayanagi M, Narisawa K, Tada K, Leisti J (1992) Identification of a common mutation in Finnish patients with nonketotic hyperglycinemia. J Clin Invest 90: 160-164

Kure S, Aoki Y, Shinka T, Sakata T, Matsubara Y, Narisawa K (1997) Dinucleotide repeat polymorphism in 65k-glutamate decarboxylase gene. Jpn J Hum Genet 42: 429-431

Lott IT, Coulombe T, Di Paolo RV, Richardson EP Jr, Levy HL (1978) Vitamin B6-dependent seizures: pathology and chemical findings in brain. Neurology 28: 47-54
Sarkar G, Sommer SS (1989) Access to a messenger RNA sequence or its protein product is not limited by tissue or species specificity. Science 244: 331-334

Scriver CR, Gibson KM (1995) Disorders of $\beta$ - and $\gamma$-amino acid in free and peptide-linked forms. In: Scriver CR, Beaudet AL, Sly WS, Valle D (eds) The metabolic and molecular bases of inherited disease, 7th edn, vol 1. McGraw-Hill, New York, pp 13491368

Scriver CR, Whelan DT (1969) Glutamic acid decarboxylase (GAD) in mammalian tissue outside the central nervous system, and its possible relevance to hereditary vitamin B6 dependency with seizures. Ann NY Acad Sciences 166: 83-96

Takahashi K, Kure S, Matsubara Y, Narisawa K (1992) Novel phenylketonuria mutation detected by analysis of ectopically transcribed phenylalanine hydroxylase mRNA from lymphoblast. Lancet 340: 1473

Yoshida T, Tada K, Arakawa T (1971) Vitamin B6-dependency of glutamic acid decarboxylase in the kidney from a patient with vitamin B6 dependent convulsion. Tohoku J Exp Med 104: 195198 